\title{
Harnessing the Electronic Health Record to Actively Support Providers with Guideline-Directed Telemetry Use
}

\author{
Maya Narayanan ${ }^{1}$ Helene Starks ${ }^{2}$ Eric Tanenbaum ${ }^{3}$ \\ Anneliese M. Schleyer ${ }^{1}$ \\ ${ }^{1}$ Department of Medicine, University of Washington, Seattle, \\ Washington, United States \\ 2 Department of Bioethics and Humanities, University of Washington, \\ Seattle, Washington, United States \\ ${ }^{3}$ Department of Internal Medicine, Washington State University College of \\ Medicine, Swedish Medical Center, Seattle, Washington, United States \\ ${ }^{4}$ Department of Quality Improvement, Harborview Medical Center, \\ Seattle, Washington, United States
}

Appl Clin Inform 2021;12:996-1001.

\author{
Ellen Robinson ${ }^{4}$ Paul R. Sutton ${ }^{1}$
}

Address for correspondence Maya Narayanan, MD, MPH, Harborview Medical Center, UW Medicine, 1959 NE Pacific Street, Box 356429, Seattle, Washington 98195, United States (e-mail: mnarayan@uw.edu).

\begin{abstract}
Keywords

- electronic health record

- telemetry

- appropriate telemetry use

Background Overuse of cardiac telemetry monitoring (telemetry) can lead to alarm fatigue, discomfort for patients, and unnecessary medical costs. Currently there are evidence-based recommendations describing appropriate telemetry use, but many providers are unaware of these guidelines.

Objectives At our multihospital health system, our goal was to support providers in ordering telemetry on acute care in accordance with evidence-based guidelines and discontinuing telemetry when it was no longer medically indicated.

Methods We implemented a multipronged electronic health record (EHR) intervention at two academic medical centers, including: (1) an order set requiring providers to choose an indication for telemetry with a recommended duration based on American Heart Association guidelines; (2) an EHR-generated reminder page to the primary provider recommending telemetry discontinuation once the guideline-recommended duration for telemetry is exceeded; and (3) documentation of telemetry interpretation by telemetry technicians in the notes section of the EHR. To determine the impact of the intervention, we compared number of telemetry orders actively discontinued prior to discharge and telemetry duration 1 year pre- to 1 year post-intervention on acute care medicine services. We evaluated sustainability at years 2 and 3 .

Results Implementation of the EHR initiative resulted in a statistically significant increase in active discontinuation of telemetry orders prior to discharge: 15\% (63.4-78.7\%) at one site and $13 \%$ at the other (64.1-77.4\%) with greater improvements on resident teams. Fewer acute care medicine telemetry orders were placed on medicine services across the system $(1,503-1,305)$ despite an increase in admissions and the average duration of telemetry decreased at both sites (62 to 47 hours, $p<0.001$ and 73 to $60, p<0.001$, respectively). Improvements were sustained 2 and 3 years after intervention.

Conclusion Our study showed that a low-cost, multipart, EHR-based intervention with active provider engagement and no additional education can decrease telemetry usage on acute care medicine services.
\end{abstract}

received

April 15, 2021

accepted after revision

September 4, 2021 (c) 2021. Thieme. All rights reserved. Georg Thieme Verlag KG,

Rüdigerstraße 14,

70469 Stuttgart, Germany
DOI https://doi.org/

10.1055/s-0041-1736338.

ISSN 1869-0327. 


\section{Background and Significance}

Cardiac telemetry monitoring in accordance with evidencebased standards predicts cardiac events, ${ }^{1}$ but inappropriate overuse leads to alarm fatigue, patient discomfort, and unnecessary resource utilization including unnecessary work-up potentially adversely impacting patient safety and increasing costs of care. ${ }^{2-4}$ The American Heart Association (AHA) has published guidelines on appropriate telemetry use $^{1}$ and the Society of Hospital Medicine's Choosing Wisely initiative recommends against using continuous telemetry monitoring outside the intensive care unit (ICU) without a protocol that governs its continuation. ${ }^{5}$ Many providers are unaware of these guidelines and recommendations and few receive education about guideline-directed telemetry use. On acute care services, telemetry is often ordered due to patients' deteriorating clinical status and less so to monitor cardiac rhythm dysfunction. ${ }^{6}$

Several studies have tried different approaches to address these shortcomings including provider education, routine feedback, provider incentives, and modifications to electronic health record (EHR) telemetry orders. These studies have demonstrated that multifaceted approaches do in fact lead to more appropriate telemetry utilization and duration of use, ${ }^{7-10}$ but may require more resources and time to complete. Other studies have shown that changes to the EHR with reminders about discontinuing telemetry via pop-up dialog boxes or automatic telemetry discontinuation after a set time period, without provider education or incentives, were enough to encourage appropriate telemetry ordering and increase timely telemetry discontinuation. ${ }^{11-13}$ To date, no study has looked at other methods using the EHR to directly ask providers to consider discontinuing telemetry when the guideline-recommended duration for a specific indication has passed.

\section{Objectives}

We describe a quality-improvement project to help physicians appropriately order telemetry and decrease unnecessary use by making modifications to the EHR. Specifically, we describe three interventions to the EHR, all of which were low cost and required minimal time to implement. We examine hours of telemetry use and percent telemetry orders discontinued prior to discharge pre- and post-intervention to determine if the EHR changes lead to improved telemetry utilization. Additionally, we look at differences in hours of telemetry use and percent telemetry orders discontinued prior to discharge between services with residents versus just hospitalists. We also examine how hours of telemetry use and percent telemetry orders discontinued change over an extended period of time ( 2 and 3 years postintervention). Our goal was to harness the EHR to actively support providers in ordering telemetry in accordance with evidence-based guidelines and discontinuing telemetry when it is no longer medically necessary on acute care services.

\section{Methods}

\section{Study Design, Setting, and Population}

The project was designed as a quality-improvement project at two academic medical centers within the University of Washington Medicine Health System, Harborview Medical Center (HMC), a 413-bed academic referral center and safety net hospital, and University of Washington Medical Center (UWMC), a 570-bed academic referral center. Data were extracted from the EHR for all hospital stays in which patients had telemetry monitored for admissions from July 2015 to June 2019. ICU telemetry usage was excluded. For patients who had telemetry ordered more than once during the same hospitalization, only the first telemetry episode was evaluated. We limited this analysis to patients cared for on acute care medicine services; some services were hospitalist only and others were teaching services with residents. Attending physicians on the resident medicine services included hospitalists and nonhospitalist internal medicine physicians. HMC has three hospitalist-run services and five resident medicine teams. UWMC has two hospitalist teams and four resident medicine teams. Patients are assigned to medicine teams based on time of day and admitting capacity of the resident teams. Each site has a distinct cardiology service.

\section{Intervention}

In July 2016, we implemented three changes to the EHR to support guideline-directed telemetry ordering without provider training or staff coaching. The first was a new telemetry order set that requires providers to use AHA-recommended indications for telemetry and lists the evidence-based duration of telemetry for each condition ( - Fig. 1). The order set includes a list of conditions for which telemetry is frequently ordered but not recommended per current standards to discourage inappropriate monitoring.

The second change involved creating an automatic paging system set up through the EHR to actively notify the primary providers caring for patients on telemetry when the order duration has exceeded the guideline-recommended duration. The page recommends discontinuation of telemetry unless there is a new indication. Pages are sent during daytime work hours and are bundled in the morning to coincide with usual rounding times. We chose direct paging of providers from the EHR as this system is updated by providers themselves identifying their role in real time. This ensures that the patient's "primary contact" who is paged is accurately identified in the context of multiple handoffs in a complex teaching environment.

The third change involved telemetry technicians moving from a paper-based record (printing telemetry strips filed in the paper chart) to documenting their findings in the EHR at one of the two sites. With implementation of the EHR, telemetry readings were the only clinical data that remained on paper except for patient registration materials, creating a gap in the EHR documentation. This shift in practice was intended to support communication between telemetry technicians, providers, and nurses and to increase provider 


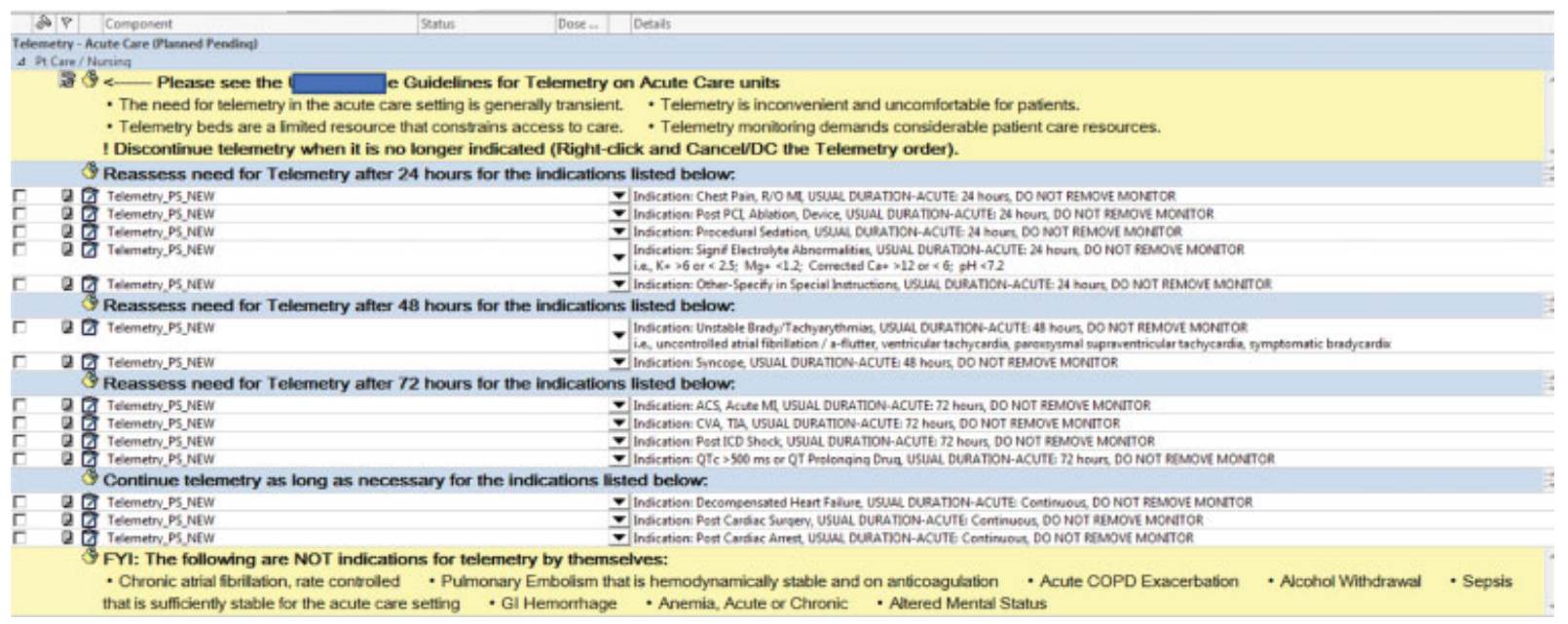

Fig. 1 Electronic health record telemetry order set requiring providers to choose an indication for telemetry with guideline-recommended duration of telemetry monitoring.

awareness of telemetry findings. The algorithm that technicians used to notify the nurse or provider of any significant changes in rhythm did not change.

\section{Data Collection and Analysis}

Data were electronically abstracted from the EHR for both hospitals for 1 year pre-intervention (July 2015-June 2016) and 1 year post-intervention (July 2016-June 2017).We reviewed two additional years of data to examine sustainability. Data including telemetry order initiation and discontinuation date and time were initially collected as part of a quality improvement project for patients admitted to medicine acute care services for whom telemetry was ordered. All identifiers except month and year of order were destroyed. Data were examined in aggregate and separately for the hospitalist-only and resident services. We also obtained the average annual patient volume and length of stay on medicine services for each hospital to control for environmental trends. At the site at which telemetry technicians transcribed documentation directly into the EHR, we surveyed them about their experience with the new workflow.

The percentage of orders actively discontinued and the average number of hours on telemetry were computed for each month and compared over time for each hospital and by service. We used $t$-tests to compare annual proportions (percent active order discontinuation) and means (hours on telemetry) for years 1, 2, and 3 against year 0 . We used Stata 16 for statistically analyses and Excel to create the run charts.

\section{Results}

Overall, this set of EHR interventions led to increased active discontinuation of telemetry monitoring on acute care medicine services with a 15\% increase at HMC (63.4-78.7\%, $p<0.001)$ and $13 \%$ increase at UWMC $(64.1-77.4 \%$, $p<0.001$; - Table 1$)$. At both sites, we saw a slightly greater increase in active discontinuation among resident teams compared with hospitalist teams (16.1 vs. 13.1\% [HMC]; 13.5 vs. $11.8 \%$ [UWMC]) and only the resident improvements were statistically significant. At HMC, residents were more likely to actively discontinue telemetry orders prior to intervention than hospitalists; this was reversed at UWMC.

In the year after implementing our EHR intervention, we also observed decreased numbers of telemetry orders across medicine services at each site ( 985 to 850 at HMC; 518 to 455 at UWMC) although the number of patients admitted to medicine services increased (-Table 1). The decrease in number of medicine acute care telemetry orders reflects

Table 1 Percentage of telemetry orders actively discontinued prior to discharge preintervention and 1 to 3 years postintervention by site and by service

\begin{tabular}{|c|c|c|c|c|c|c|c|}
\hline \multirow[t]{2}{*}{ Telemetry discontinued } & \multirow{2}{*}{$\begin{array}{l}\text { Baseline } \\
\text { Pre: 2015-2016 } \\
\%(n)\end{array}$} & \multicolumn{2}{|c|}{$\begin{array}{l}\text { Year } 1 \\
\text { post: } 2016-2017\end{array}$} & \multicolumn{2}{|c|}{$\begin{array}{l}\text { Year } 2 \\
\text { post: } 2017-2018\end{array}$} & \multicolumn{2}{|c|}{$\begin{array}{l}\text { Year } 3 \\
\text { post: } 2018-2019\end{array}$} \\
\hline & & $\%(n)$ & $\begin{array}{l}\text { \% change } \\
\text { from baseline }\end{array}$ & $\%(n)$ & $\begin{array}{l}\text { \% change } \\
\text { from baseline }\end{array}$ & $\%(n)$ & $\begin{array}{l}\text { \% change } \\
\text { from baseline }\end{array}$ \\
\hline Site 1: all services & $63.4(985)$ & $78.7(850)$ & $15.3^{\mathrm{a}}$ & $79.7(883)$ & $16.3^{\mathrm{a}}$ & $79.8(952)$ & $16.4^{\mathrm{a}}$ \\
\hline Resident services & $64.2(801)$ & $80.3(669)$ & $16.1^{\mathrm{a}}$ & $78.7(666)$ & $14.5^{\mathrm{a}}$ & $76.4(750)$ & $12.2^{\mathrm{a}}$ \\
\hline Hospitalist services & $59.8(184)$ & $72.9(181)$ & $13.1^{\mathrm{a}}$ & $83.8(167)$ & $24.0^{\mathrm{a}}$ & $78.2(202)$ & $18.4^{\mathrm{a}}$ \\
\hline Site 2: all services & $64.1(518)$ & $77.4(455)$ & $13.3^{\mathrm{a}}$ & $73.7(475)$ & $9.6^{a}$ & $75.8(532)$ & $11.7^{\mathrm{a}}$ \\
\hline Resident services & $63.0(408)$ & $76.5(340)$ & $13.5^{\mathrm{a}}$ & $75.8(356)$ & $12.8^{\mathrm{a}}$ & $76.0(384)$ & $13.0^{\mathrm{a}}$ \\
\hline Hospitalist services & $68.2(110)$ & $80.0(115)$ & $11.8^{\mathrm{a}}$ & $67.2(119)$ & -1.0 & $75.0(148)$ & 6.8 \\
\hline
\end{tabular}

${ }^{a} p \leq 0.01$. 
Table 2 Mean hours of telemetry use preintervention and 1 to 3 years postintervention, by site and by service

\begin{tabular}{|c|c|c|c|c|c|c|c|}
\hline \multirow[t]{2}{*}{ Telemetry hours } & \multirow{2}{*}{$\begin{array}{l}\text { Baseline } \\
\text { Pre: } 2015-2016 \\
\text { Mean (SD) } \\
{[95 \% \text { Cl] }}\end{array}$} & \multicolumn{2}{|c|}{$\begin{array}{l}\text { Year } 1 \\
\text { post: } 2016-2017\end{array}$} & \multicolumn{3}{|c|}{$\begin{array}{l}\text { Year } 2 \\
\text { post: } 2017-2018\end{array}$} & \multirow{2}{*}{$\begin{array}{l}\text { Year } 3 \text { post: } \\
2018-2019 \\
\text { Mean change } \\
\text { from baseline }\end{array}$} \\
\hline & & $\begin{array}{l}\text { Mean (SD) } \\
{[95 \% \mathrm{CI}]}\end{array}$ & $\begin{array}{l}\text { Mean change } \\
\text { from baseline }\end{array}$ & $\begin{array}{l}\text { Mean (SD) } \\
{[95 \% \mathrm{CI}]}\end{array}$ & $\begin{array}{l}\text { Mean change } \\
\text { from baseline }\end{array}$ & $\begin{array}{l}\text { Mean (SD) } \\
{[95 \% \mathrm{CI}]}\end{array}$ & \\
\hline Site 1: all services & $\begin{array}{l}62.0(81.9) \\
{[56.8-67.1]}\end{array}$ & $\begin{array}{l}47.0(51.5) \\
{[43.6-50.5]}\end{array}$ & $14.9^{\mathrm{a}}$ & $\begin{array}{l}46.8(46.2) \\
{[43.7-50.0]}\end{array}$ & $15.1^{\mathrm{a}}$ & $\begin{array}{l}53.9(59.0) \\
{[50.2-57.7]}\end{array}$ & $8.0^{\mathrm{b}}$ \\
\hline Resident services & $\begin{array}{l}65.1(87.9) \\
{[59.0-71.2]}\end{array}$ & $\begin{array}{l}49.0(55.0) \\
{[44.8-53.2]}\end{array}$ & $16.1^{\mathrm{a}}$ & $\begin{array}{l}47.9(48.6) \\
{[44.2-51.6]}\end{array}$ & $17.2^{\mathrm{a}}$ & $\begin{array}{l}56.5(61.2) \\
{[52.1-60.9]}\end{array}$ & $8.6^{\mathrm{b}}$ \\
\hline Hospitalist services & $\begin{array}{l}48.4(45.0) \\
{[41.8-54.9]}\end{array}$ & $\begin{array}{l}39.9(34.5) \\
{[34.8-44.9]}\end{array}$ & $8.5^{b}$ & $\begin{array}{l}42.4(35.1) \\
{[37.1-47.8]}\end{array}$ & 6.0 & $\begin{array}{l}44.4(48.9) \\
{[37.6-51.1]}\end{array}$ & 4.0 \\
\hline Site 2: all services & $\begin{array}{l}73.3(88.7) \\
{[65.6-81.0]}\end{array}$ & $\begin{array}{l}59.8(68.0) \\
{[53.5-66.1]}\end{array}$ & $13.5^{\mathrm{a}}$ & $\begin{array}{l}58.3(68.0) \\
{[52.2-64.5]}\end{array}$ & $15.0^{\mathrm{a}}$ & $\begin{array}{l}60.4(64.7) \\
{[54.9-65.9]}\end{array}$ & $12.9^{a}$ \\
\hline Resident services & $\begin{array}{l}75.2(89.0) \\
{[66.5-83.8]}\end{array}$ & $\begin{array}{l}59.1(69.3) \\
{[51.7-66.5]}\end{array}$ & $16.1^{\mathrm{a}}$ & $\begin{array}{l}58.2(64.6) \\
{[51.5-64.9]}\end{array}$ & $16.9^{\mathrm{a}}$ & $\begin{array}{l}61.7(63.9) \\
{[55.3-68.1]}\end{array}$ & $13.4^{\mathrm{b}}$ \\
\hline Hospitalist services & $\begin{array}{l}66.4(87.7) \\
{[49.8-83.0]}\end{array}$ & $\begin{array}{l}62.0(64.4) \\
{[50.1-73.9]}\end{array}$ & 4.4 & $\begin{array}{l}58.6(77.7) \\
{[44.5-72.7]}\end{array}$ & 7.8 & $\begin{array}{l}57.3(66.6) \\
{[46.4-68.1]}\end{array}$ & 9.2 \\
\hline
\end{tabular}

Abbreviations: $\mathrm{Cl}$, confidence interval; SD, standard deviation.

${ }^{\mathrm{a}} p \leq 0.01$.

${ }^{\mathrm{b}} \mathrm{p} \leq 0.05$.

fewer telemetry orders among resident teams. Numbers of telemetry orders were similar among hospitalists in the 2 years. There was also a statistically significant decrease in mean duration of telemetry at both sites (62 to 47 hours, $p<0.001$ at HMC; 73.3 to 59.8 hours, $p<0.001$ at UWMC) despite essentially unchanged hospital length of stay on medicine services (-Table 2). Decreases in telemetry duration were only statistically significant among resident teams.

In years 2 and 3 postintervention, the percentage of telemetry orders actively discontinued prior to discharge continued to be higher than preintervention levels overall (pre: $63.4 \%, 1$ year post: $78.7 \%, 2$ years post: $79.7 \%, 3$ years post: $79.8 \%$ ) and for hospitalist and resident services at HMC (-Table 1). At UWMC, the percentage of active order discontinuation remained higher than baseline in years 2 and 3 after intervention overall (pre: $64.1 \%, 1$ year post: $77.4 \%$, 2 years post $73.7 \%, 3$ years post $75.8 \%$ ) and among resident teams; the percent increase was highest in the first year after intervention. Among hospitalists, there was greater variability in years 2 and 3 after intervention. An improved lower average duration of telemetry episodes was sustained in years 2 and 3 compared with preintervention baselines at both sites on all teams (- Table 2).

At both sites, rates of active discontinuation of telemetry orders and decreased telemetry duration trended toward improvement, although there was significant month-tomonth variation in practice (-Fig. 2).

At HMC where telemetry technicians documented telemetry findings directly in the EHR, 10 surveys were completed. Overall, technicians were very satisfied with this initiative. In

\section{A. Percent Active Order Discontinuation}

\section{B. Mean Telemetry Duration (hours)}
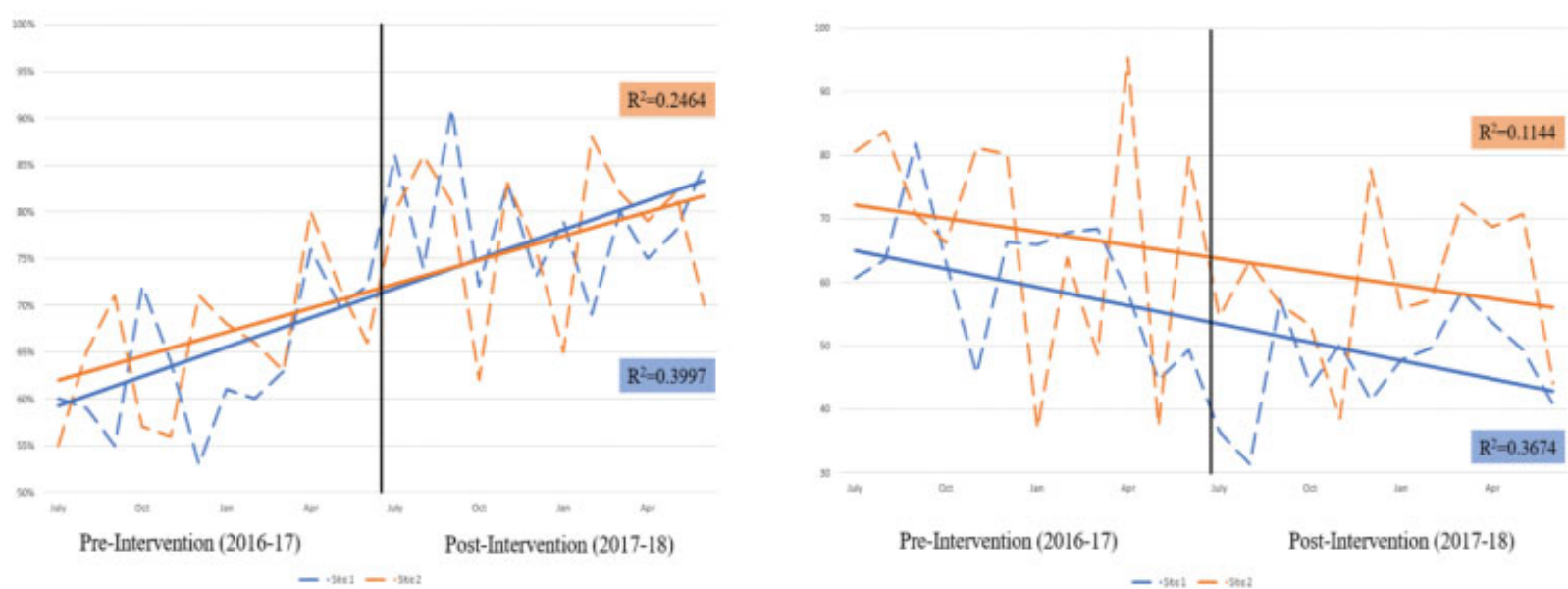

Fig. 2 Telemetry ordering practices by month for all medicine services by hospital (site $1=$ HMC, site 2 =UWMC). (A) A greater percentage of telemetry orders was discontinued before hospital discharge after the EHR intervention but there was significant month-to-month variation at both hospitals. (B) Trend of average hours of telemetry use shows a decrease in average hours of use over time at both hospitals with significant month-to-month variation. EHR, electronic health record; HMC, Harborview Medical Center; UWMC, University of Washington Medical Center. 
total, $89 \%$ agreed/strongly agreed that the initiatives improved interdisciplinary team communication and $88 \%$ agreed that increased teamwork improved their job satisfaction. One technician stated, "I feel like telemetry technicians are communicating with doctors and nurses to improve patient care."

\section{Discussion}

Harnessing the EHR to support guideline-directed telemetry ordering improved telemetry utilization on acute care medicine services at two academic referral hospitals in our health system with fewer telemetry orders despite increased medicine admissions, increased active discontinuation of telemetry orders prior to discharge, and decreased average duration of telemetry. Impact was greatest on resident services although hospitalist ordering practices also showed some improvements. Improvements were sustained over time.

One strength of this intervention was employing the EHR to assist in clinical decision-making in real time. Formal education on guideline-directed telemetry use is often absent or lacking in residency programs and medical schools. Harnessing capabilities built into the EHR to support guideline-directed ordering and actively discontinuation of telemetry not only provides a persistent effect without the need for specific training, but also does so at minimal cost.

Of the three arms of our intervention, the direct page to the patient's primary physician recommending discontinuation of telemetry unless a new indication existed was felt to be the most impactful. In a cluster-randomized clinical trial, Najafi and colleagues demonstrated that a targeted EHR alert or "pop-up" dialog box as a single-component intervention significantly reduced telemetry monitoring duration by 8.7 hours per hospitalization on an academic general medicine service. ${ }^{12}$ These findings were similar to improvements seen in our study on the hospitalist services; however, we saw greater improvements with average decrease per hospitalization of 16 to 17 hours on medicine resident services that were sustained in year 2 after intervention. Year 3 performance continued to show an improvement over baseline. Our study is unique in that we used a text page to engage the primary contact provider and not a pop-up alert. Text pages are a routine method of active inpatient communication. In complex academic medical centers with medicine patients bedded in multiple units, the direct page is an active message that supports clinical decision-making in real time and that reaches providers regardless of where they are in the hospital. Studies have shown that pop-up alerts also lead to alert fatigue and may be dismissed given their overuse. ${ }^{14}$ Other studies have shown that multicomponent interventions including education, process change, routine feedback, and financial incentives decrease telemetry utilization. ${ }^{7}$ As in the Najafi et al study, our intervention was not significantly resource-intensive and only involved modifications to the EHR. Directly engaging the primary provider for the patient to consider discontinuation of telemetry orders also supported active-provider decision-making for patients whose telemetry order exceeded the standard duration for a specific indication. Studies have shown that automatic discontinuation of telemetry also decreases telemetry utilization..$^{10,11}$ However, this approach requires nurses to contact physicians if discontinuation is believed to be unsafe according to an algorithm. In our intervention, the provider can proactively make this decision which in turn supports clinical decisionmaking as well as resident learning in our academic centers. This intervention may be particularly effective at academic centers as we noted a greater impact on resident teaching services. At both hospitals, the resident services had a statistically significant increase in the amount of telemetry orders discontinued prior to discharge and average duration of telemetry. This may reflect a lower level of baseline knowledge of guideline-directed telemetry use among residents and increased reliance on order-set recommendations and suggestions sent by active page.

We hypothesized that technician entry of telemetry findings directly into the notes section of the EHR notes would have a significant impact and we observed higher rates and greater improvements in active discontinuation of telemetry orders prior to discharge at the site that employed this intervention compared with the site that did not. This may represent a greater awareness of patients who remained on telemetry as the findings were in the patients' charts with other clinical information. We did not, however, see significantly greater decreases in duration of telemetry episodes. Nonetheless, telemetry technicians were satisfied with the changes and reporting improved intra-team communication and job experience.

Overall, there were improved practices in years 2 and 3 at both sites relative to pre-intervention baseline, suggesting that a structural improvement such as an EHR intervention can have a sustained effect.

\section{Limitations}

It is possible that providers, particularly residents, ordered telemetry less often because they had improved knowledge of when telemetry should be ordered based on the order-set indications or because they felt the telemetry order set was cumbersome. Our analysis showed that there was significant month-to-month variation in practice suggesting that additional unmeasured factors, such as telemetry indication, differences in patient population, or monthly census variation that we could not account for, may have contributed to changed practice patterns. Nonetheless our interventions were implemented in a real-world setting and the total telemetry usage on acute care medicine was lower. Since we included only initial telemetry orders, our data are biased against those situations in which telemetry was restarted. We were not able to capture any adverse impacts of earlier discontinuation of telemetry monitoring although there were no incident reports filed at either site related to earlier telemetry discontinuation and no code blues. We did not collect readmission information for medicine patients whose telemetry orders were included in this review; however, overall medicine service readmission rates did not increase over this time period. 


\section{Conclusion}

Our study showed that a low-cost, multipart, EHR-based intervention including a text page directly to the responsible provider with no additional education decreased telemetry usage and that the intervention had more effect on resident services. As many institutions adopt EHRs, such EHR interventions may support effective use of telemetry in acute care medicine.

\section{Clinical Relevance Statement}

Changing provider behavior is difficult. The EHR system can be harnessed to actively assist physicians in choosing guideline-directed medical interventions and limiting unnecessary use. Additionally, innovations using the EHR are often cheaper and less time-consuming than other interventions.

\section{Multiple Choice Questions}

1. Disadvantages of cardiac telemetry monitoring include:

a. Prolonged hospitalization.

b. Increased risk of infection.

c. Alarm fatigue.

d. Can only be performed at academic medical centers.

Correct Answer: The correct answer is c. Disadvantages of cardiac telemetry include alarm fatigue, unnecessary medical costs, and patient discomfort. In and of itself, telemetry is not known to prolong hospitalization or cause infections, and it is used in both community and academic medical centers.

2. Which of the following changes were made to the University of Washington EHR to help providers limit unnecessary telemetry use?

a. Pop-up reminder generated by the EHR to consider discontinuing telemetry if no longer medically necessary.

b. An automatic page through the EHR to discontinue telemetry if no longer medically necessary.

c. Automatic discontinuation of the telemetry order by the EHR when telemetry use has exceeded guidelinerecommended use.

d. Required daily attestation in the EHR by the provider stating the necessity of telemetry use.

Correct Answer: The correct answer is b, as described in the Methods section. Choices a, c, and d were not implemented.

Protection of Human and Animal Subjects

The University of Washington Human Subjects reviewed our application and determined that our study does not involve "human subjects" as defined by federal regulation and that it does not require exempt status or institutional review board review. Animal subjects were not included in this research.

Conflict of Interest

None declared.

\section{References}

1 Drew BJ, Califf RM, Funk M, et al; American Heart Association Councils on Cardiovascular Nursing, Clinical Cardiology, and Cardiovascular Disease in the Young. Practice standards for electrocardiographic monitoring in hospital settings: an American Heart Association scientific statement from the Councils on Cardiovascular Nursing, Clinical Cardiology, and Cardiovascular Disease in the Young: endorsed by the International Society of Computerized Electrocardiology and the American Association of Critical-Care Nurses. Circulation 2004;110(17):2721-2746

2 Knight BP, Pelosi F, Michaud GF, Strickberger SA, Morady F. Clinical consequences of electrocardiographic artifact mimicking ventricular tachycardia. N Engl J Med 1999;341(17):1270-1274

3 Benjamin EM, Klugman RA, Luckmann R, Fairchild DG, Abookire SA. Impact of cardiac telemetry on patient safety and cost. Am J Manag Care 2013;19(06):e225-e232

4 Chen DW, Park R, Young S, Chalikonda D, Laothamatas K, Diemer G. Utilization of continuous cardiac monitoring on hospitalist-led teaching teams. Cureus 2018;10(09):e3300-e3310

5 Bulger J, Nickel W, Messler J, et al. Choosing wisely in adult hospital medicine: five opportunities for improved healthcare value. J Hosp Med 2013;8(09):486-492

6 Chaudhary R, Kingsley T, Sarkar S, et al. Telemetry: appropriateness of initial assignment and duration in nonintensive setting. Am J Manag Care 2020;26(11):459-460

7 Edholm K, Kukhareva P, Ciarkowski C, et al. Decrease in inpatient telemetry utilization through a system wide electronic health record change and a multifaceted hospitalist intervention. J Hosp Med 2018;13(08):531-536

8 Chahine J, Thapa B, Gosai F, et al. Interventions to decrease overuse of cardiac monitoring (telemetry) when transitioning from the intensive care until to the regular nursing floor. Cureus 2019;11(03):e4311

9 Stoltzfus KB, Bhakta M, Shankweiler C, Mount RR, Gibson C. Appropriate utilisation of cardiac telemetry monitoring: a quality improvement project. BMJ Open Qual 2019;8(02):e000560

10 Pendharkar SS, Barry IB, Patibandla S, et al. AHA telemetry guidelines improve telemetry utilization in the inpatient setting. Am J Manag Care 2020;26(11):476-481

11 Dressler R, Dryer MM, Coletti C, Mahoney D, Doorey AJ. Altering overuse of cardiac telemetry in non-intensive care unit settings by hardwiring the use of American Heart Association guidelines. JAMA Intern Med 2014;174(11):1852-1854

12 Rizvi W, Munguti CM, Mehta J, Kallail KJ, Youngman D, Antonios S. Reducing over-utilization of cardiac telemetry with pop-ups in an electronic medical record system. Cureus 2017;9(05): e1282-e1285

13 Najafi N, Cucina R, Pierre B, Khanna R. Assessment of a targeted electronic health record intervention to reduce telemetry duration: a cluster-randomized clinical trial. JAMA Intern Med 2019; 179(01):11-15

14 Wright A, Aaron S, Seger DL, Samal L, Schiff GD, Bates DW. Reduced effectiveness of interruptive drug-drug interaction alerts after conversion to a commercial electronic health record. J Gen Intern Med 2018;33(11):1868-1876 